\title{
Galactic TeV Gamma Ray Sources and Diffuse Emission - Results from Milagro
}

\author{
Petra Hüntemeyer ${ }^{1}$ for the Milagro Calibration \\ Michigan Technological University \\ 1400 Townsend Drive, Houghton, MI 49931, USA \\ E-mail: petra@mtu.edu
}

The Milagro Observatory collected data between the years 1999 and 2008. The original detector was a 60 by $80 \mathrm{~m}$ pond of up to $8 \mathrm{~m}$ deep water instrumented with 723 PMTs that were arranged in two layers. The PMTs measured the Cherenkov light that relativistic particles of extensive air showers produce when they traverse the water. In 2004, 173 water tanks were added surrounding the pond and covering an area of 200 by $200 \mathrm{~m}$. The addition of this array of water tanks approximately doubled the sensitivity of the detector. Milagro detected multiple sources of TeV gamma-rays, most are close to the Galactic plane and all are coincident with bright $\mathrm{GeV}$ pulsars. In addition, after accounting for the emission from these sources, there remains a significant excess emission in the Galactic plane which may be due to diffuse emission produced by cosmic rays interacting with matter and radiation in the galaxy or due to unresolved sources. Here, the results that are based on an analysis of the complete Milagro data set that has a peak sensitivity at energies of $10 \mathrm{TeV}$ to $50 \mathrm{TeV}$ will be summarized. Moreover, ongoing analyses that exclusively utilize combined data from both detector components, the central pond and the tank array will be discussed. Finally, the Milagro results will be compared to measurements of other gamma ray telescopes as well as to model predictions, and implications of these comparisons will be discussed.

1 Petra Hüntemeyer 
5th Texas Symposium on Relativistic Astrophysics -TEXAS2010

Heidelberg, Germany

December $06-112010$ 


\section{Introduction}

The Milagro experiment [1] was a first generation water Cherenkov detector designed to measure charged particles from extensive air showers that are caused by $\mathrm{TeV}$ gamma rays. It was located in the Jemez Mountains near Los Alamos, New Mexico, at an elevation of $2630 \mathrm{~m}$ and at $106.60^{\circ} \mathrm{W}$ longitude and $35.88^{\circ} \mathrm{N}$ latitude. The experiment collected data from the Northern Hemisphere sky between years 2001 and 2008. The detector consisted of two main components: A central pond of water and an array of tanks filled with water surrounding the former. The central pond was instrumented with two layers of photo multipliers, a deep layer, below $6 \mathrm{~m}$ of water, called "muon layer" and a shallow layer, below $1.5 \mathrm{~m}$ of water, called "air shower layer". The muon layer contained 273 Hamamatsu R5912 photomultiplier tubes (PMT), the air shower layer contained 450 PMTs of the same type. In 2004, the array of 173 water tanks, measuring $2.4 \mathrm{~m}$ in diameter and $1 \mathrm{~m}$ in height, each instrumented with a down-ward facing PMT, was added. Including this so-called "outrigger" array the effective area of the detector was about $30,000 \mathrm{~m}^{2}$.

The results presented in this paper are based on the complete data set of the Milagro observatory. Over the years, the detector simulation has continuously improved based on extensive systematic studies that deepened the understanding of the detector behavior. Changes in the detector response over time are taken into account and the gamma-hadron separation algorithm is optimized accordingly. In addition, an energy reconstruction technique has been developed, allowing for a spectral analysis of point and extended $\mathrm{TeV}$ gamma-ray sources.

In the following, the results from three studies of galactic $\mathrm{TeV}$ gamma rays using data collected with the Milagro Observatory will be summarized and discussed:

1. A measurement of Multi-TeV emission from Galactic sources in the Fermi LAT bright source list

2. A spectral analysis of Galactic sources.

3. A status report of the diffuse $\mathrm{TeV}$ gamma-ray emission from the galactic plane.

\section{Measurement of Multi-TeV Emission from Galactic Sources in the Fermi Bright Source List (BSL)}

In 2009, the Milagro collaboration presented a survey of galactic sources in the Fermi bright source catalog using the complete data the experiment collected between 2001 and 2008 [2]. Of the $205 \mathrm{GeV}$ sources in the Fermi BSL 34 are in the field of view of the Milagro observatory (i.e. have a declination of $>-5^{0}$ ) and are possibly Galactic. Sixteen of the 34 sources are pulsars, five are co-located with supernova remnants, one with an x-ray binary, and twelve sources are un-identified. At 14 out of the 34 locations TeV excesses of greater than 3 standard deviations were observed. Figure 1 shows a Milagro map of the galactic plan and 
exploded views of all the locations (except for the Crab pulsar wind nebula). The chance probability of measuring significances of $>3 \sigma$ at 13 out of the 34 locations is only $10^{-17}$.
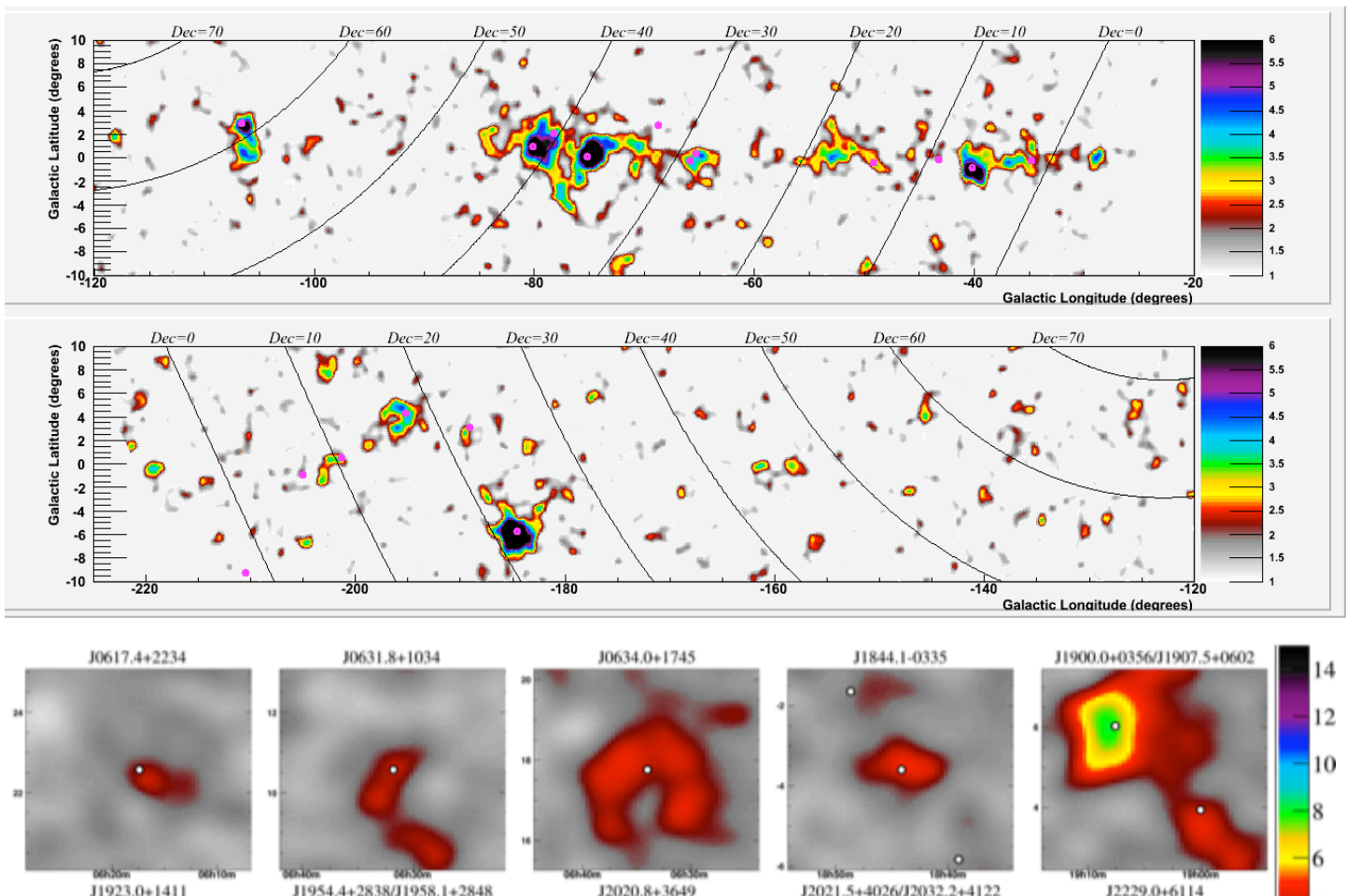
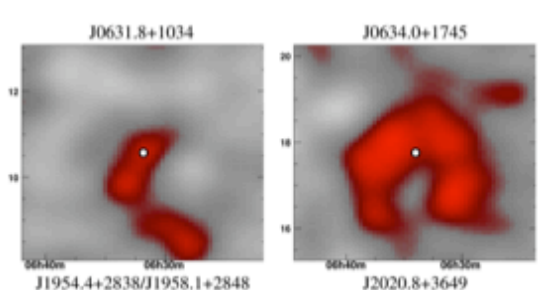

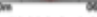
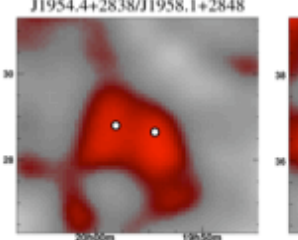

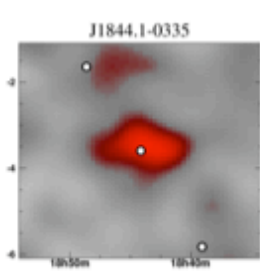

J2021.5+4026/32032.2+4122

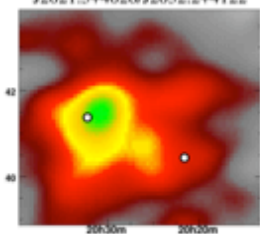

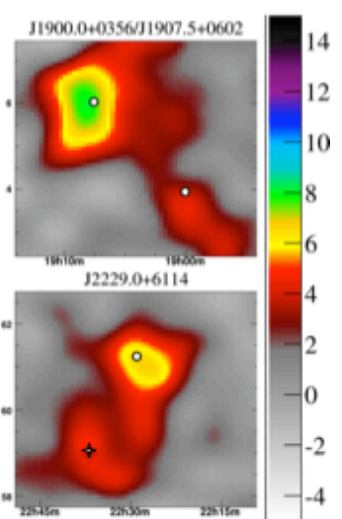

Figure 1 Upper plot: Milagro map of significances in the Galactic plane, Fermi BSL locations are shown as magenta dots; lower plot: exploded views of the 3 locations in the Milagro maps, the white dots represent the corresponding Fermi BSL source locations, the crosses represent the associtations with Fermi pulsars that have been discovered since the publication of [2].

Since the publication of the BSL survey by Milagro, the Fermi satellite has discovered eight additional pulsars in Milagro's field of view, two of which are associated with significant excesses in Milagro data. They are also shown - as crosses - in Figure 1. One association is seen in the Cygnus region, where the Milagro experiment measures a significance of $4.5 \sigma$ at the location of a newly detected Fermi pulsar. The second association is seen in the region of the "Boomerang" pulsar wind nebula with a significance of $4.7 \sigma$ at the location of the newly detected Fermi pulsar. In summary, eleven of the $16 \mathrm{GeV}-\mathrm{TeV}$ associations seen in the Milagro and Fermi data are co-located with pulsars, three are co-located with super nova remnants, and two with un-identified GeV sources. All the brightest Milagro excesses in the Galactic plane are coincident with $\mathrm{GeV}$ pulsars. 


\section{Spectral Analysis of Galactic Sources}

In order to reconstruct the spectrum of the brightest $\mathrm{TeV}$ sources that Milagro detected an energy parameter has been developed based on extensive Monte Carlo studies. It has been found that the sum of the fraction of air shower PMTs and of outrigger PMTs that are triggered by an air shower,

$$
\mathcal{F}=\frac{N_{A S}}{N_{A S}^{l i v e}}+\frac{N_{O R}}{N_{O R}^{\text {live }}}
$$

(aka FrASOR) is strongly correlated with the primary energy of the air shower and hence a good measure of it. The Milagro data is divided into nine bins of FraSOR between 0.2 and 2, where each bin has a size of 0.2. Assuming a generalized spectral shape with an exponential cut-off,

$$
\frac{d N}{d E}\left(I_{o}, \alpha, E_{\text {cut }}\right)=I_{o}\left(\frac{E}{20 T e V}\right)^{-\alpha} \exp \left(\frac{-E}{E_{\text {cut }}}\right)
$$

the expected event excess in each bin is calculated for a range of values of $I_{0}$, $\alpha$, and $E_{\text {cut }}$ using Monte Carlo simulations. The best spectral fit is then found by minimizing

$$
\chi^{2}\left(I_{o}, \alpha, E_{\text {cut }}\right)=\sum_{j=\text { epochs } i=\text { FrASOR bins }} \frac{\left(P_{i, j}\left(I_{o}, \alpha, E_{\text {cut }}, \text { Declination }\right)-M_{i, j}\right)^{2}}{\sqrt{\delta P_{i, j}^{2}+\delta M_{i, j}^{2}}} .
$$

where $j$ denotes different epochs or phases of Milagro data taking. The resulting best fit of the Crab Nebula spectrum is shown in Figure 2 and compared with a measurement by H.E.S.S [3]. Figure 2 shows excellent agreement between the H.E.S.S. and Milagro measurements, a confirmation of the energy analysis of Milagro and of the validity of the simulation of the Milagro instrument sensitivity.

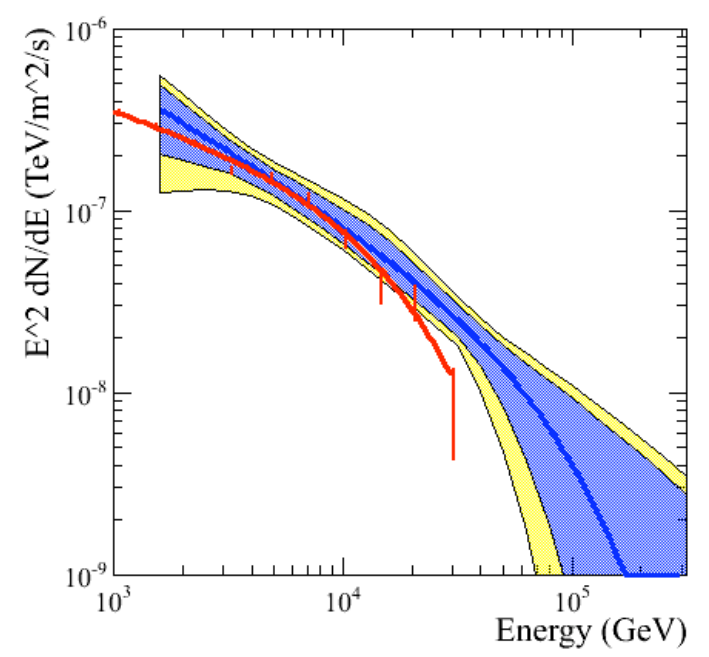

Figure 2 The spectrum of the Crab Nebula as measured by Milagro. The one (blue) and two (yellow) $\sigma$ regions of the best fit are shown as shaded bands. The H.E.S.S. fit to the Crab Nebula is overlaid in red [3]. 


\section{Diffuse TeV Gamma-Ray Emission from the Galactic Plane}

In 2008, Milagro published a measurement of diffuse $\mathrm{TeV}$ gamma-ray emission from four separate regions of the Galactic plane [4]. In two regions, the Cygnus region $\left(-2^{0}<b<2^{0}\right.$, $\left.65^{0}<1<85^{0}\right)$ and the Inner Galaxy $\left(\left(-2^{0}<\mathrm{b}<2^{0}, 30^{0}<1<65^{0}\right)\right.$ a slight excess with respect to the cosmic ray propagation model GALPROP optimized to match the diffuse $\mathrm{GeV}$ gamma-ray flux as measured by EGRET was observed. The recent measurements by Fermi LAT [5] at intermediate latitudes contradict the EGRET result and are strong evidence that the optimized model is incorrect. Figure 3 shows a comparison of the Milagro diffuse differential flux measurement at a median energy of $15 \mathrm{TeV}$ with predictions of the most recent version of the GALPROP model, vs. 54 [6]. A flux of $7.5 \times$ the prediction is observed in the Cygnus region and $3.7 \times$ the prediction is observed from the region along the Galactic ridge.
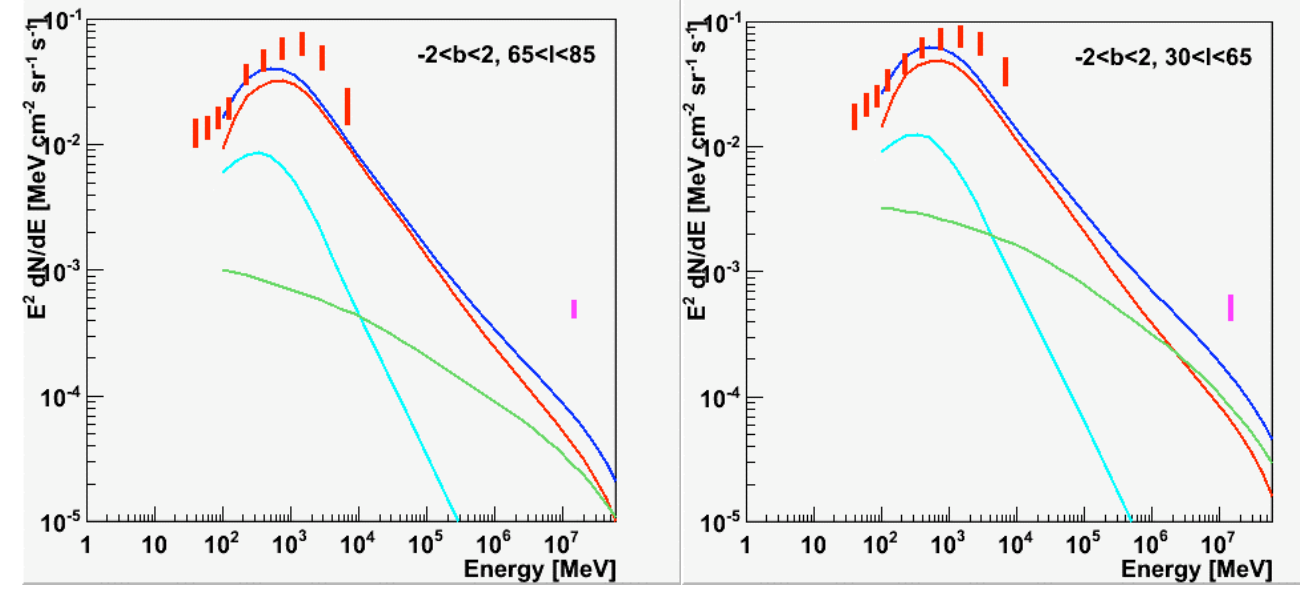

Figure 3: Milagro diffuse TeV for the Cygnus region (left) and the "inner Galaxy" (right) (magenta point) compared to the conventional GALPROP model, vs. 54. The red points represent the measured EGRET fluxes. The predicted total flux is shown in blue, the pion contribution in red, the IC contribution in green, and the bremsstrahlungs contribution in light blue. At Fermi LAT energies the spectrum is well described by a power-law with the spectral index of Galactic cosmic rays. Note that at Milagro energies the IC contribution becomes more important according to GALPROP predictions.

Even though dark matter annihilation has been put forward as an explanation for this excess [7], the large Milagro flux is likely an overestimate of diffuse emission due to the presence of unresolved localized accelerators. For example, there may be weak sources such as pulsar wind nebulae that the previous Milagro analysis was not sensitive enough to identify and therefore were not subtracted.

\section{Conclusion}

The results of three different studies of Galactic gamma rays with Milagro data have been presented. In a survey of the galactic sources in the Fermi BSL it is found that all of the brightest Milagro sources are spatially coincident with Fermi pulsars. Furthermore, a new energy reconstruction algorithm has been developed for the spectral analysis of Milagro data and the resulting best fits to the Crab Nebula data are in good agreement with IACT 
measurements. Finally, significant diffuse excess with respect to the cosmic ray propagation model GALPROP is observed in the Cygnus region and in the inner Galaxy. The HAWC experiment (currently under construction in Mexico) with its improved sensitivity will help to resolve this discrepancy and most likely increase the number $\mathrm{TeV}$ source detections.

\section{References}

[1] R. Atkins et al., TeV Gamma-Ray Survey of the Northern Hemisphere Sky Using the Milagro Observatory, 2004, ApJ, 608, 680.

[2] A.A. Abo et al., Milagro Observations of Multi-TeV Emission from Galactic Sources in the Fermi Bright Source List, 2009, ApJ, 700, L127.

[3] F. Aharonian et al., Observations of the Crab Nebula with HESS, 2006, AAP, 457, 899.

[4] A.A. Abdo et al., A Measurement of the Spatial Distribution of Diffuse TeV Gamma-Ray Emission from the Galactic Plane with Milagro, 2008, ApJ, 688, 1078.

[5] Abdo, A. A., et al., Fermi Large Area Telescope Measurements of the Diffuse Gamma-Ray Emission at Intermediate Galactic Latitudes, 2009, PRL, 103, 251101.

[6] Strong, A. W., private communication.

[7] Bi, X.-J., Chen, T.-L., Wang, Y., Yuan, Q., The Diffuse GeV-TeV $\gamma$-Ray Emission of the Cygnus Region, 2009, ApJ, 695, 883. 